\title{
QUANTUM MECHANICAL STUDIES ON THE EFFECT OF INTERMOLECULAR ROTATION OF THE STACKING INTERACTION OF DIAZINE ISOMERS
}

\author{
Mahasweta Choudhury, Shruti Sharma, Benzir Ahmed \\ and Bipul Bezbaruah* \\ Department of Applied Sciences, Gauhati University, Guwahati-781014, Assam, India. \\ *E-mail: bipulbezbaruah@gmail.com
}

\begin{abstract}
Non-covalent interaction plays an important role in different $\pi$-system, aromatic compounds and other biomolecules. The $\pi-\pi$ stacking interaction between heteroaromatic systems is an important area of research. Herein, we have taken one simple organic heterocycle such as a diazine molecule and some of its isomers to investigate the $\pi-\pi$ stacking interaction. Stacking interactions of heterocycles are highly sensitive towards the position, bond angle and dihedral angle of the heteroatoms and it also results in different interactions for different stacked models. We have chosen the stacked dimers of diazine isomers (1,2-diazine, 1,3-diazine and 1,4-diazine) and quantum mechanical studies have been carried out to compute the $\pi-\pi$ stacking interaction using (Møller-Plesset) MPn methods. The diazine isomers show variation in stacking interaction with different intermolecular rotation and we observed a conformational variation in different stacked models of diazine.
\end{abstract}

Keywords: MPn, Diazine, Dihedral Angle, $\pi-\pi$ Stacking etc.

(C) RASĀYAN. All rights reserved

\section{INTRODUCTION}

The non-covalent interactions are important due to their role in the structure and function of supramolecular systems in various areas of chemistry, material science, catalysis, biology, etc. The noncovalent interactions include hydrogen bonding, ion-dipole, dipole-dipole, $\pi-\pi$, van der Waals, metalligand interactions. ${ }^{1-2}$ Among the various non-covalent interactions the $\pi-\pi$ stacking interaction between heteroaromatic systems has gained the utmost attention in recent years due to its ubiquitous nature in many biological and supramolecular systems. This interaction plays a key role in determining the overall assembly and packing of these systems. ${ }^{3-4}$ The $\pi-\pi$ interaction among the aromatic rings plays a crucial role in stabilizing the double-helical structure of DNA and RNA and protein structures. ${ }^{5-7}$ The $\pi-\pi$ interactions are equally important in many material science applications such as in carbon nanotubes, graphene, electronic properties of organic molecules having $\pi$-conjugated systems. ${ }^{8-10}$

Heterocyclic molecules are very much important, e.g. naturally occurring indole derivatives have several pharmacological activities. ${ }^{11-13}$ Due to the electronegativity difference between the carbon (C) and the heteroatom (X) in the C-X bond of heterocycles, the polarization of the electron cloud takes place and thus these molecules show better stacking interaction compared to non-heterocyclic counterpart. Nitrogen containing heterocycles are the most important one to study for non-covalent interactions, as it shows maximum electron charge density on it. They show better stacking interaction between themselves as the heteroatom plays an important role in the non-covalent interaction. Sponer and Hobza had studied the stacking interaction in dimers of various nitrogen-containing heterocycles using MP2 level of theory. ${ }^{14}$ Mignon and co-workers studied the interactions in mixed dimers of pyridine and substituted benzene. Tsuzuki and co-workers studied the interaction of thiophene molecules in several orientations. ${ }^{15}$ Sathyamurthy et al. has done a detailed study on the stacking interaction of dimers of pyridine, pyrazine and tetrazine molecule in different orientations. ${ }^{16-19}$ The present work is aimed to study the stacking

Rasayan J. Chem., 13(1), 663-671(2020)

http://dx.doi.org/10.31788/RJC.2020.1315535

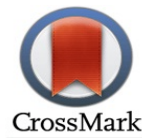


RASĀYAN J. Chem.

Vol. 13 | No. 1 |663 - 671| January - March | 2020

interaction in homodimers (12-12, 13-13 and 14-14 diazine) of three isomers of diazine (1,2 diazine, 1,3 diazine and 1,4 diazine) in a different orientation with intermolecular rotation. We also investigated the stacking interactions for the heterodimers (12-13, 13-14 and 12-14 diazine) of diazine isomers. The results would allow us to have a better understanding of the non-covalent interaction of nitrogen containing heterocycles in organic and inorganic systems.

\section{EXPERIMENTAL}

Initially, all the diazine and its isomers were constructed and then optimized. Further, these optimized geometries were used for constructing various stacked models of diazine isomers with the help of the JoinMolecule package of software. Also, Arguslab was also used to visualize and observe the different stacked models. For studying the long-range non-covalent interaction such as $\pi-\pi$ interaction, the quantum mechanical ab initio method is the most reliable one. All the stacked models have been computed by using MPn (Møller-Plesset) method with GaussView5.0 and Gaussian09 software packages. MP2/6$311++\mathrm{G}(\mathrm{d}, \mathrm{p})$ basis set was used for the optimization of models and the same basis set was used for the calculation of single point energy.

Diazine dimers can be directly stacked like sandwich structure with different dihedral angles (intermolecular rotation), but in our observations, we have taken only $0^{\circ}, 60^{\circ}, 120^{\circ}$ and $180^{\circ}$ dihedral angles, which show preferable stacking interaction among them. Here, $0^{\circ}$ dihedral angle can be considered as eclipsed and the rest of them i.e. $60^{\circ}, 120^{\circ}$ and $180^{\circ}$ are considered as staggered conformations. The $\pi-\pi$ stacking interaction energies for the stacked models of diazine isomers can be calculated by the following equation:

$$
\text { Interaction Energy }=\mathrm{E}_{\text {stacked }}-2 \mathrm{E}_{\text {single }}
$$

Where, $E_{\text {stacked }}=$ Single point energy of the stacked diazine molecule and $E_{\text {single }}=$ Single point energy of the single unstacked diazine molecule. All the calculations were computed by using Gaussian09. ${ }^{20}$

\section{RESULTS AND DISCUSSION}

The current work describes the non-covalent $\pi-\pi$ stacking interactions among the diazine isomers both homo and hetero stacked models. Before making the stacked models, the three diazine isomers $(1,2-$ diazine, 1,3-diazine and 1,4-diazine) were optimized by using MP2 level of theories in Gaussian09 software package (Fig.-1).

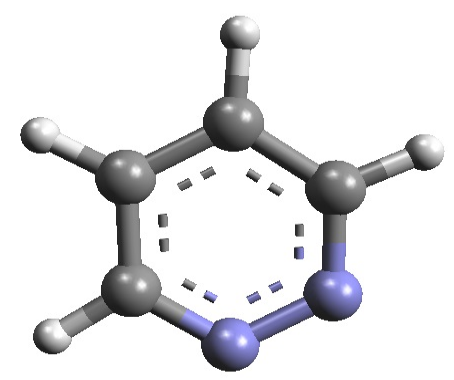

(a)

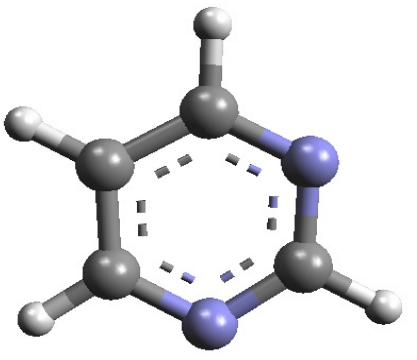

(b)

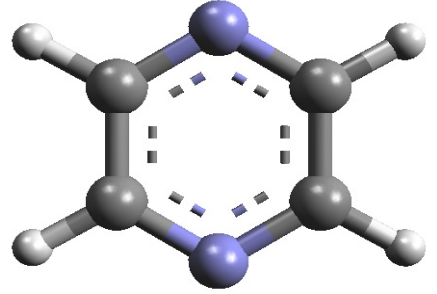

(c)

Fig.-1: Optimized models of Diazine isomers (a) 1,2Diazine, (b) 1,3Diazine and (c) 1,4Diazine

During the construction of stacked models of diazine dimers the intermolecular separation was kept constant at $3.6 \AA$, which was taken as the standard intermolecular distance for any long-range noncovalent interaction. The stacking interactions of diazine stacked dimers have been studied by horizontal shifting of one ring above others (keeping the second ring at the constant position), both in the positive and negative direction along any axis. Herein, the horizontal shifting for diazine dimers was done along the $\mathrm{X}$-axis from 0 to $+3 \AA$ and 0 to $-3 \AA$ directions. All the stacked models of diazine stacked dimers were 
RASĀYAN J. Chem.

Vol. 13 | No. 1 |663 - 671| January - March | 2020

studied in the gas phase. Moreover, the models for diazine dimers were also constructed by rotating one ring at different rotations keeping the other ring fixed. Here, we calculated the stacking interactions for diazine dimers with different intermolecular rotations (dihedral angle), the preferable intermolecular rotations are viz. $0^{\circ}, 60^{\circ}, 120^{\circ}$ and $180^{\circ}$ (Fig.-2).

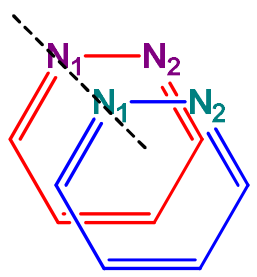

$0^{\circ}$

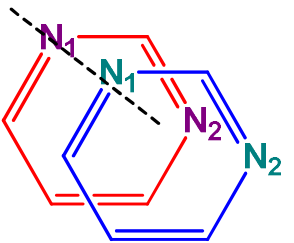

$0^{\circ}$

Intermolecular rotation of 1,2-Diazine dimer

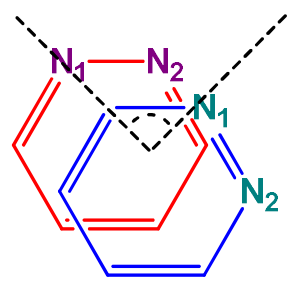

$60^{\circ}$

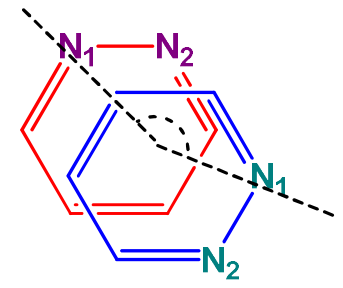

$120^{\circ}$

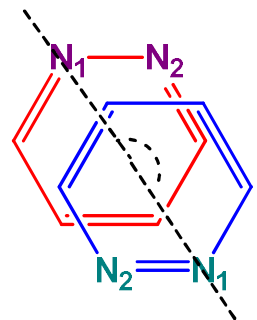

$180^{\circ}$

Intermolecular rotation of 1,3-Diazine dimer

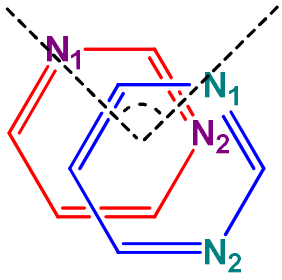

$60^{\circ}$

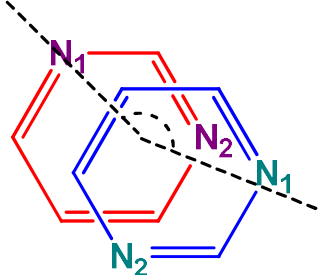

$120^{\circ}$

Intermolecular rotation of 1,4-Diazine dimer

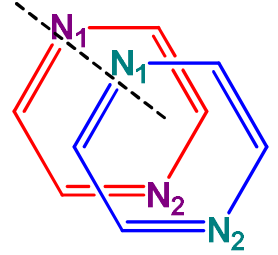

$0^{\circ}$

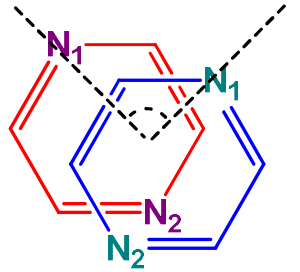

$60^{\circ}$

Fig.- 2: Pictorial Representation of Intermolecular Rotation for Stacked Diazine Dimmer at $0^{\circ}, 60^{\circ}, 120^{\circ}$ and $180^{\circ}$

It is well known that more is the negative interaction energy value of the staked model more is the stability of the stacked model which results in the most stable and favored conformers for any stacked model. The minimum interaction energy can easily be reflected in the interaction of energy plots. Therefore, the minimum points of the interaction energy $(\mathrm{kcal} / \mathrm{mol})$ versus horizontal shifting $(\AA)$ plot shows the most negative and most favored structure (Figs.-3 to 6). In our investigations, the most negative value of stacking interaction energy for 1,2-diazine dimer (homo stacked dimer), has been computed as $6.395 \mathrm{kcal} / \mathrm{mol}$, which is for intermolecular rotation of $180^{\circ}$ at a horizontal shifting of $+1.0 \AA$ (Table 1 ). In a 1,2-diazine stacked system, as we increase the intermolecular rotation (dihedral angle) from $0^{\circ}$ to $180^{\circ}$ for different stacked models, the interaction energy also becomes more negative and it gives the most stable conformation for $180^{\circ}$ rotation (Fig.-3 and 7). Thus, for the 1,2-diazine dimer system the stability for stacked models increases in the order $0^{\circ}<60^{\circ}<120^{\circ}<180^{\circ}$. Similar calculations were also carried out for 1,3- and 1,4-diazine of homo stacked dimer systems. In 1,3- homo stacked dimer system $0^{\circ}$ intermolecular rotation is almost equivalent to $180^{\circ}$, therefore we carried out a calculation for the stacking interactions with $0^{\circ}, 60^{\circ}$ and $120^{\circ}$ intermolecular rotations. 
RASĀYAN J. Chem.

Vol. 13 | No. 1 |663 - 671| January - March | 2020

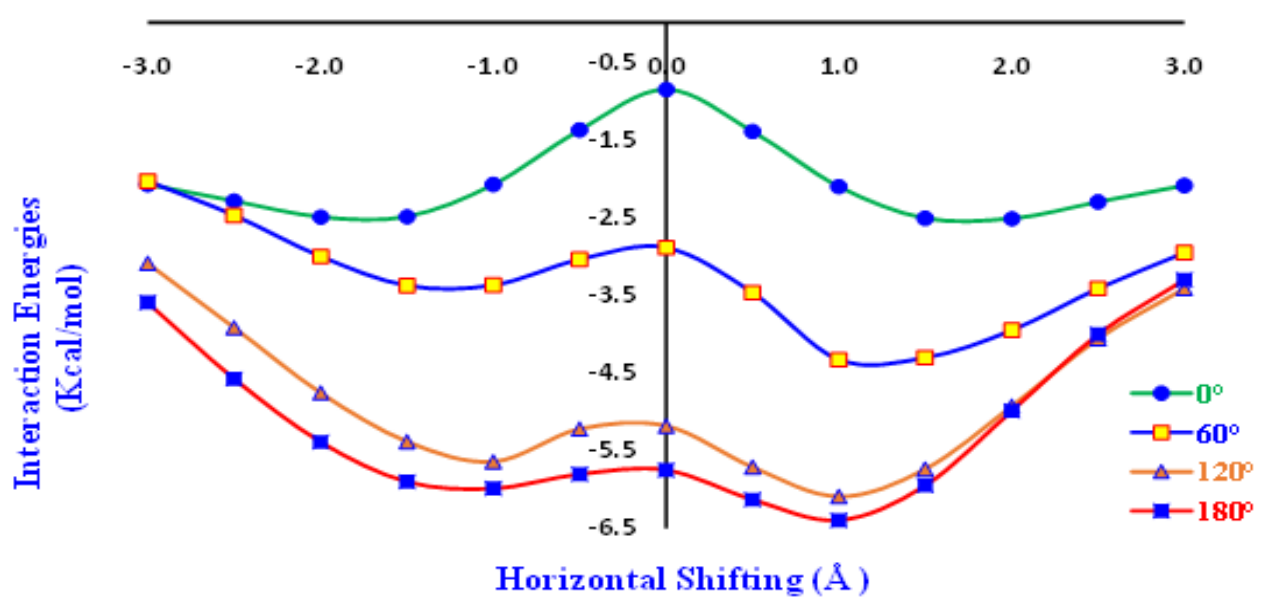

Fig.-3: Plot of Interaction Energies (kcal/mol) Versus Horizontal Shifting $(\AA)$ for 1,2-1,2 Diazine Dimer

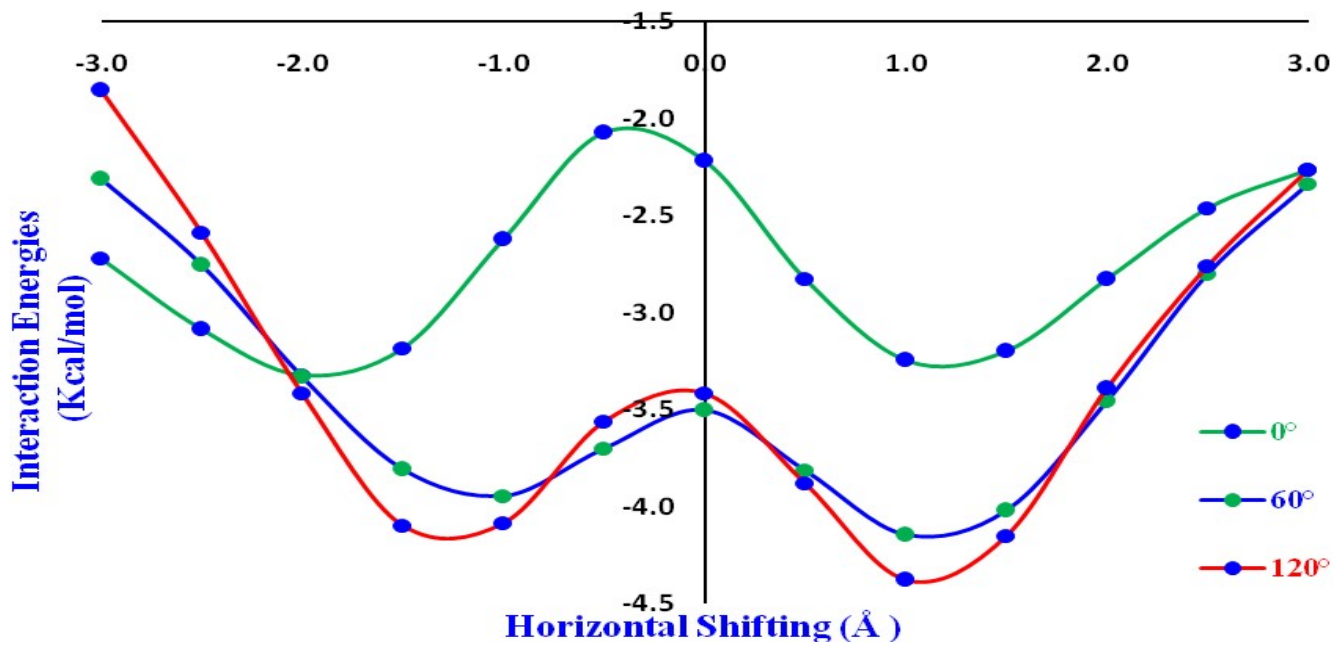

Fig.-4: Plot of Interaction Energies (kcal/mol) Versus Horizontal Shifting $(\AA)$ for 1,3-1,3 Diazine Dimer

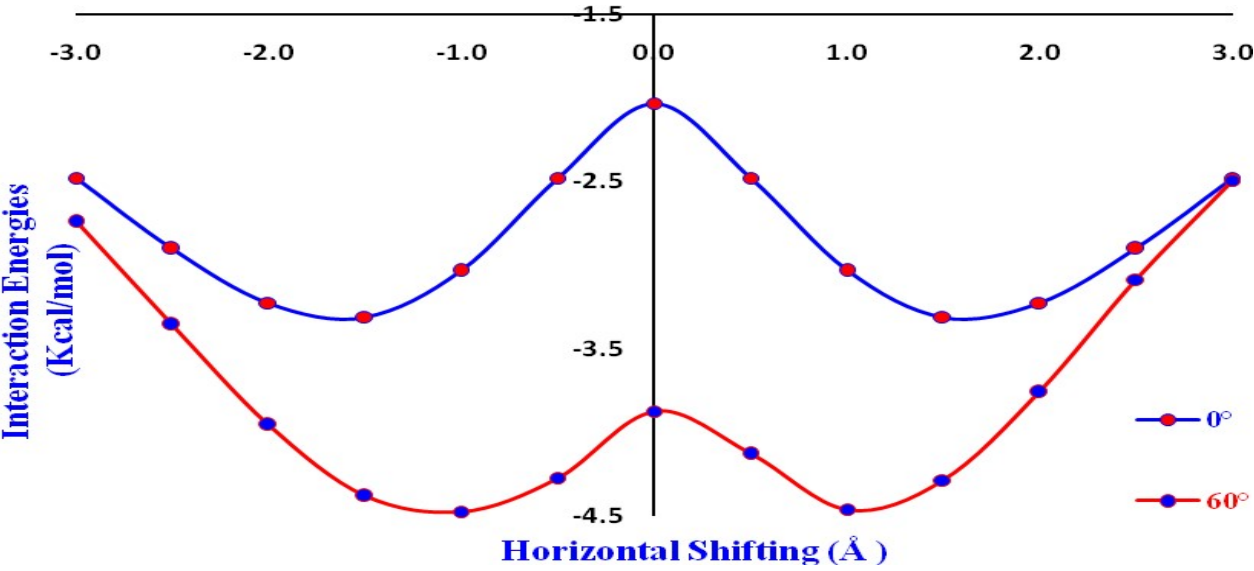

Fig.-5: Plot of Interaction Energies (kcal/mol) Versus Horizontal Shifting $(\AA)$ for 1,4-1,4 Diazine Dimer 
RASĀYAN J. Chem.

Vol. 13 | No. 1 |663 - 671| January - March | 2020

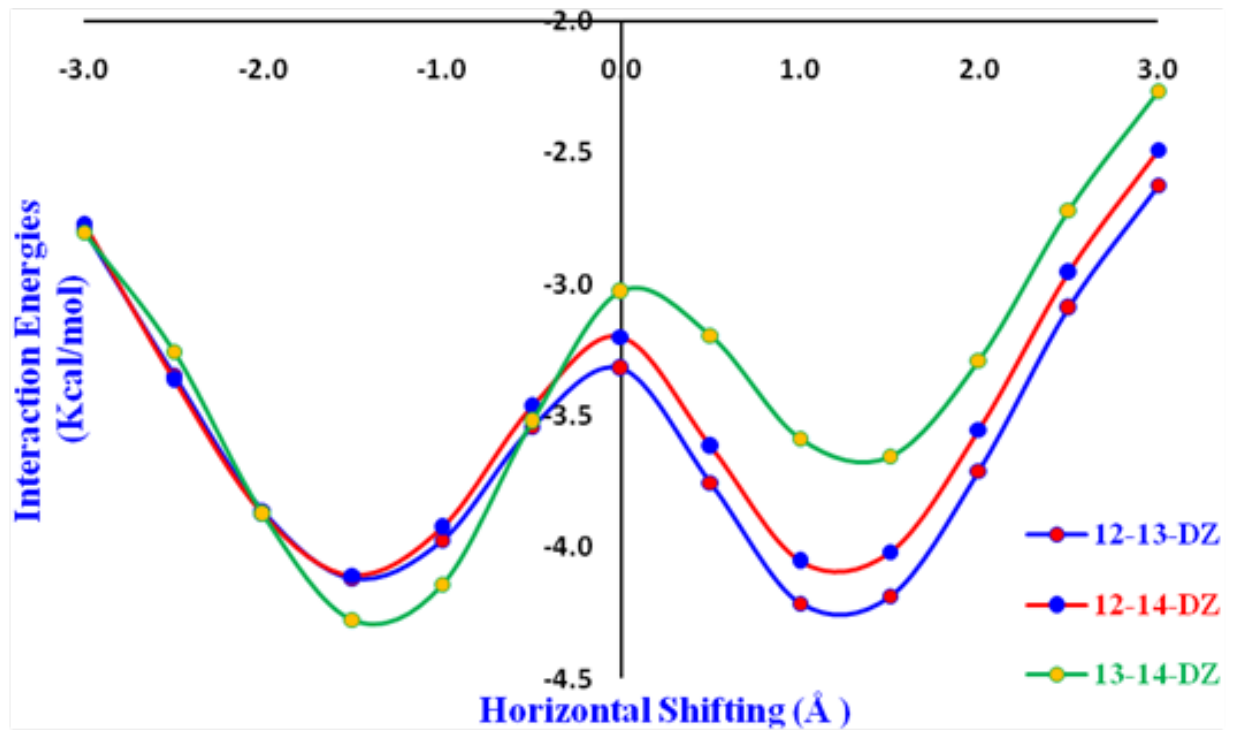

Fig.-6: Plot of Interaction Energies (kcal/mol) Versus Horizontal Shifting $(\AA)$ for Hetero mixed Dimers 1,2-1,3, 1,21,4 and $13-14$
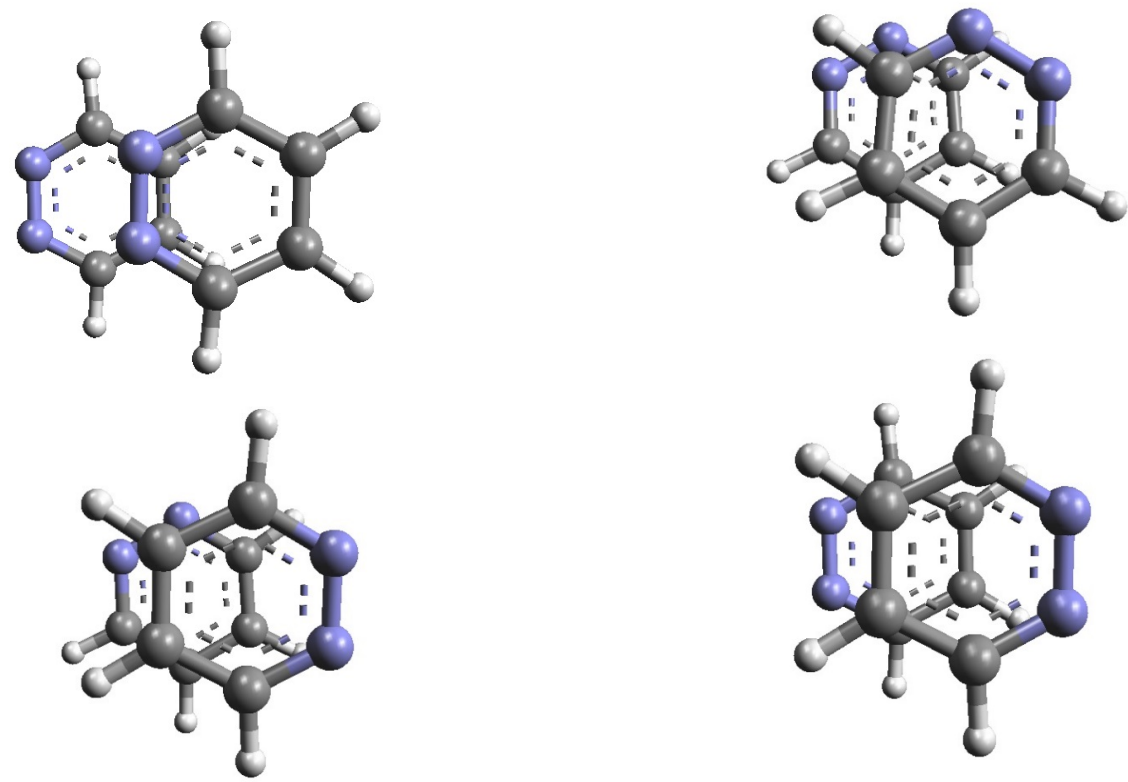

Fig.-7: Minimized Stacked Models of 1,2 Diazine Stacked Dimers with (a) $0^{\circ}$, (b) $60^{\circ}$, (c) $120^{\circ}$ and (d) $180^{\circ}$ Intermolecular Rotation

Here, the most stable stacking interaction energy value for the stacked dimer models are computed as $4.379 \mathrm{kcal} / \mathrm{mol}$ for the intermolecular molecular rotation of $120^{\circ}$, at $+1.0 \AA$ horizontal shifting (Fig. -4 and 8 ). This trend of stability is observed due to the repulsion between the nitrogen atoms of the diazine ring which decreases as we increase the intermolecular rotation of the diazine isomers. But, the repulsion is maximum and gives less negative value in the interaction energy plots when there is no horizontal shifting of the diazine rings and without intermolecular rotation (Figs.- 3 to 6 ).

On the other hand, since the 1,4-diazine system has purely symmetric stacked conformers, therefore in a 1,4-diazine stacked dimer system, $0^{\circ}$ and $60^{\circ}$ intermolecular rotations are equivalent to $120^{\circ}$ and $180^{\circ}$ respectively. Hence, we carried out a calculation for the stacking interactions with only $0^{\circ}$ and $60^{\circ}$ 
RASĀYAN J. Chem.

Vol. 13 | No. 1 |663 - 671| January - March | 2020

intermolecular rotations. In the 1,4-diazine homo dimer system, the most stable interaction was observed at $60^{\circ}$ intermolecular rotation with interaction energy value $-4.474 \mathrm{kcal} / \mathrm{mol}$ at $-1.0 \AA$ horizontal shifting (Fig.-5 and 9). The overall trend of stability order for all the stacked homo dimer models of diazine isomers is almost the same, i.e. increases the stability with an increase in the intermolecular rotation.

Encouraged by the $\pi-\pi$ stacking interaction of homo stacked dimers of different diazine isomers, we further studied the stacking interaction of hetero stacked diazine isomers or mixed diazine isomers. For this we have chosen to study the stacking interaction among 1,2-1,3, 1,3-1,4 and 1,2-1,4 hetero stacked diazine dimer at a normal favored position without any intermolecular rotations and vertical separation

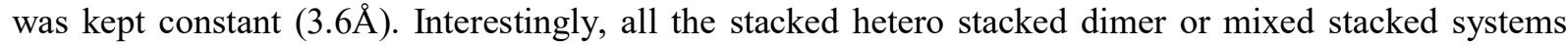
$(1,2-1,3 ; 1,3-1,4$ and $1,2-1,4)$ are comfortably stacked within them, as we have calculated the interaction energy (Fig.- 6 and 10). The computed stacking interaction energies for 1,2-1,4; 1,3-1,4 and 1,3-1,4diazine stacked heterodimers were found as $-4.212,-4.106$ and $-4.275 \mathrm{kcal} / \mathrm{mol}$ respectively as shown in Table 1. But, the most negative interaction energy value is found for 1,3-1,4 stacked dimeric systems i.e. $4.275 \mathrm{kcal} / \mathrm{mol}$, it happens because of the repulsion between the nitrogen atoms of the two rings is minimum. The actual sequence of stability of the stacking interaction of diazine hetero stacked dimers is found to be; $1,2-1,3<1,2-1,4<1,3-1,4$ diazine dimer.

We also computed the Mulliken charge density of the diazine isomers using MP2/6-311++G(d,p) $\mathrm{POP}=\mathrm{NPA}$ basis set, for both unstacked diazine molecule as well as stacked diazine molecules for the most stable stacked conformers. The Mulliken charge density shows the change in net charge density at the $\mathrm{N}$ atoms of the diazine ring for unstacked as well as stacked diazine molecules, i.e. when the difference in change of charge density at the $\mathrm{N}$ atoms are more, the stacked model will be more stable (Table-2 and 3).
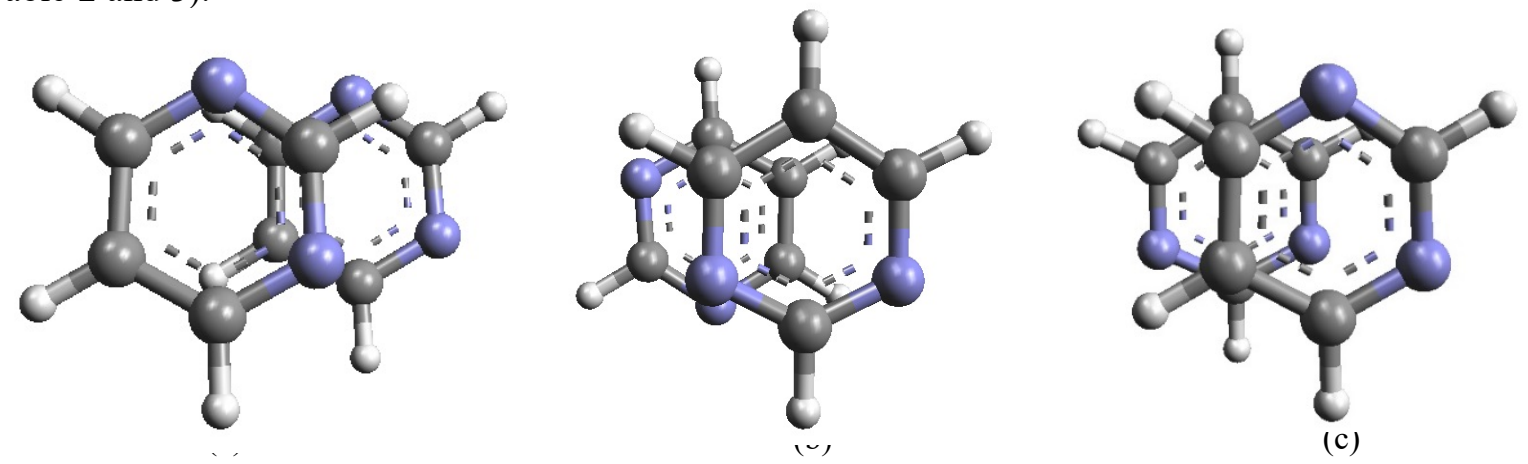

Fig.- 8: Minimized Stacked Models of 1,3 Diazine Stacked Dimers with (a) $0^{\circ}$ (b) $60^{\circ}$ and (c) $120^{\circ}$ Intermolecular Rotation
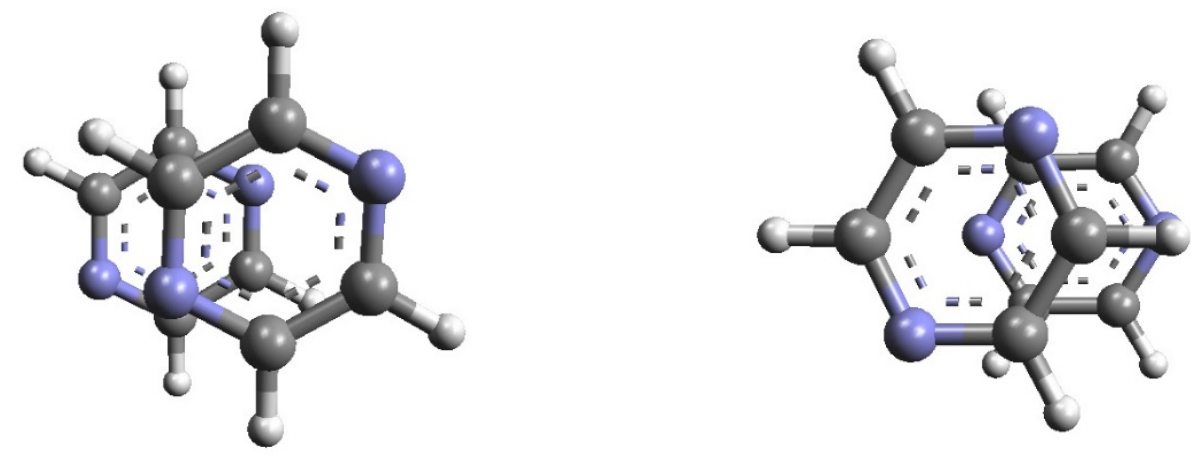

Fig.-9: Minimized Stacked Models of 1,4 Diazine Stacked Dimers with (a) $0^{\circ}$ and (b) $60^{\circ}$ Intermolecular Rotation 
RASĀYAN J. Chem.

Vol. 13 | No. 1 |663 - 671| January - March | 2020

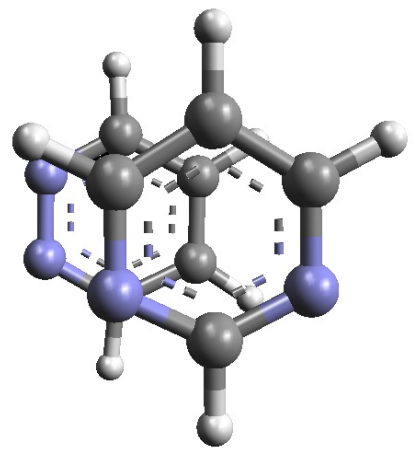

(a)

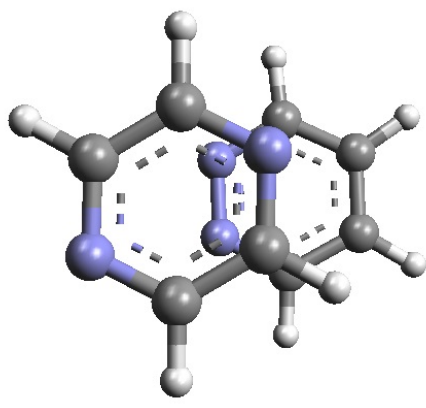

(b)

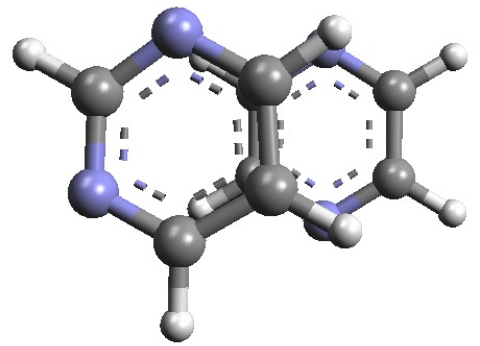

(c)

Fig.-10: Minimized Stacked Models of mixed Diazine Stacked Dimer (a) 1,2-1,3 (b) 1,2-1,4 and (c) $1,3-1,4$

Table-1: Stacking Interaction Energies ( $\mathrm{kcal} / \mathrm{mol})$ for minimized Diazine Dimer stacked Models (MP2/6-311++G(d,p))

\begin{tabular}{c|c|c|c}
\hline \multirow{2}{*}{ Stacked Models } & $\begin{array}{c}\text { Intermolecular } \\
\text { rotation }\end{array}$ & $\begin{array}{c}\text { Horizontal } \\
\text { Shifting }(\AA)\end{array}$ & $\begin{array}{c}\text { Interaction Energies } \\
(\mathrm{kcal} / \mathrm{mol})\end{array}$ \\
\hline \multirow{3}{*}{ 12-12-Diazine } & $0^{\circ}$ & +2.0 & -2.517 \\
\cline { 2 - 4 } & $60^{\circ}$ & +1.0 & -4.330 \\
\cline { 2 - 4 } & $120^{\circ}$ & +1.0 & -6.091 \\
\cline { 2 - 4 } & $180^{\circ}$ & +1.0 & -6.395 \\
\hline \multirow{3}{*}{ 13-13-Diazine } & $0^{\circ}$ & -2.0 & -3.323 \\
\cline { 2 - 4 } & $60^{\circ}$ & +1.0 & -4.146 \\
\cline { 2 - 4 } & $120^{\circ}$ & +1.0 & -4.379 \\
\hline \multirow{2}{*}{ 14-14-Diazine } & $0^{\circ}$ & +1.5 & -3.310 \\
\cline { 2 - 4 } & $60^{\circ}$ & -1.0 & -4.474 \\
\hline 12-13-Diazine & $0^{\circ}$ & +1.0 & -4.212 \\
\hline 12-14-Diazine & $0^{\circ}$ & -1.5 & -4.106 \\
\hline 13-14-Diazine & $0^{\circ}$ & -1.5 & -4.275 \\
\hline
\end{tabular}

Table-2: Computed Mulliken Charges for minimized Diazine Dimer Stacked Models $(\mathrm{MP} 2 / 6-311++\mathrm{G}(\mathrm{d}, \mathrm{p}) \mathrm{POP}=\mathrm{NPA})$

\begin{tabular}{c|c|c|c|c|c|c|c}
\hline \multirow{2}{*}{ Models } & $\begin{array}{c}\text { Intermolecular } \\
\text { rotation }\end{array}$ & \multicolumn{2}{|c|}{ Ring (Unstacked) } & \multicolumn{2}{c|}{ Ring 1 (stacked) } & \multicolumn{2}{c}{ Ring 2 (stacked) } \\
\cline { 2 - 7 } & $0^{\circ}$ & -0.0541 & -0.0541 & -0.0239 & -0.0380 & 0.0396 & -0.0156 \\
\hline \multirow{3}{*}{$\begin{array}{c}12-12- \\
\text { Diazine }\end{array}$} & $60^{\circ}$ & -0.0541 & -0.0541 & 0.0176 & -0.0084 & -0.0890 & 0.1457 \\
\cline { 2 - 7 } & $120^{\circ}$ & -0.0541 & -0.0541 & -0.0383 & 0.0257 & -0.0952 & 0.1468 \\
\cline { 2 - 7 } & $180^{\circ}$ & -0.0541 & -0.0541 & -0.0293 & 0.0169 & 0.0083 & -0.0239 \\
\hline \multirow{2}{13-13-}{} & $0^{\circ}$ & -0.2165 & -0.2166 & -0.0445 & -0.0311 & -0.1032 & -0.1562 \\
\cline { 2 - 8 } Diazine & $60^{\circ}$ & -0.2165 & -0.2166 & -0.1423 & -0.1203 & -0.0930 & -0.0906 \\
\cline { 2 - 8 } & $120^{\circ}$ & -0.2165 & -0.2166 & -0.1327 & -0.1394 & -0.0604 & -0.1381 \\
\hline 14-14- & $0^{\circ}$ & -0.1148 & -0.1148 & -0.0193 & -0.0447 & -0.0170 & -0.0409 \\
\cline { 2 - 8 } Diazine & $60^{\circ}$ & -0.1148 & -0.1148 & -0.0391 & -0.0598 & -0.0203 & -0.0425 \\
\hline
\end{tabular}


RASĀYAN J. Chem.

Vol. 13 | No. 1 |663 - 671| January - March | 2020

Table -3: Computed Mulliken Charges for minimized mixed Diazine Dimer Stacked Models $(\mathrm{MP} 2 / 6-311++\mathrm{G}(\mathrm{d}, \mathrm{p}) \mathrm{POP}=\mathrm{NPA})$

\begin{tabular}{c|c|c|c|c|c|c|c|c}
\hline \multirow{2}{*}{$\begin{array}{c}\text { Diazine } \\
\text { Models }\end{array}$} & \multicolumn{4}{|c|}{ Unstacked Models } & \multicolumn{4}{c}{ Stacked models } \\
\cline { 2 - 8 } & \multicolumn{2}{|c|}{ Ring 1 } & \multicolumn{2}{c|}{ Ring 2 } & \multicolumn{2}{c}{ Ring 1 } & \multicolumn{2}{c}{ Ring 2 } \\
\cline { 2 - 8 } & $\mathrm{N} 1$ & $\mathrm{~N} 2$ & $\mathrm{~N} 3$ & $\mathrm{~N} 4$ & $\mathrm{~N} 1$ & $\mathrm{~N} 2$ & $\mathrm{~N} 3$ & $\mathrm{~N} 4$ \\
\hline $12-13$ & -0.0541 & -0.0541 & -0.2165 & -0.2166 & 0.0132 & -0.0155 & -0.0772 & -0.0718 \\
\hline $12-14$ & -0.0541 & -0.0541 & -0.1148 & -0.1148 & -0.0508 & -0.1852 & -0.0107 & -0.0367 \\
\hline $13-14$ & -0.2165 & -0.2166 & -0.1148 & -0.1148 & -0.0508 & -0.1852 & -0.0107 & -0.0376 \\
\hline
\end{tabular}

\section{CONCLUSION}

In this study, we have investigated the quantum mechanical MP2 study on the $\pi-\pi$ stacking interaction of nitrogen containing heterocycles, viz. different isomers of diazine molecules. From the above results, we observed that the diazine isomers stacks nicely and shows effective $\pi-\pi$ stacking interactions at different intermolecular rotations. The most negative interaction energy is computed for the most stable minimized stacked conformer for each intermolecular rotation. The above results reveal that the diazine stacked dimer shows less negative interaction energy, gives a highly repulsive stacked structure when the intermolecular rotation is $0^{\circ}$. From the interaction energy plots, we have observed that the stability of stacked models of diazine isomers varies with intermolecular rotation, i.e 1,2-diazine stacked dimer highly stabilizes at an intermolecular rotation of $180^{\circ}$ and shows the most favoured stacking interactions, while for 1,3- and 1,4-diazine dimer stabilizes at an intermolecular rotation of $120^{\circ}$ and $60^{\circ}$, gives the most favoured stacking interaction.

\section{ACKNOWLEDGMENT}

Authors are highly grateful to the AICTE-TEQIP-3 fund and Ministry of Human Resource Development (MHRD), New Delhi, for providing research assistance.

\section{REFERENCES}

1. T. Dahl, Acta Chemical Scandinavica, 48, 95(1994), DOI:10.3891/acta.chem.scand.48-0095

2. C.G. Classens, J.F. Stoddart, Journal of Physical Organic Chemistry, 10(5), 254(1997), DOI: 10.1002/(SICI)1099-1395(199705)10:5<254::AID-POC875>3.0.CO;2-3

3. E.A. Meyer, R.K. Castellano and F. Diedriech, Angewandte Chemie Inernational Edition, 42(11),1210(2003), DOI:10.1002/anie.200390319

4. T.J. Mooibroek, P. Gamez, Inorgamica Chimica Acta, 360(1), 381(2007), DOI: 10.1016/j.ica.2006.07.061

5. W. Saenger, Principles of Nucleic Acid Structure, Springer, New York, (1984).

6. S.K. Burley and G.A. Petsko, Science, 229(4708), 23(1985), DOI:10.1126/science.3892686

7. C.A. Hunter, J. Singh and J.M. Thronton, Journal of Molecular Biology, 218(4), 837(1991), DOI: $10.1016 / 0022-2836(91) 90271-7$

8. T.C. Dinadayalane, L. Grob, T. Simeon and H. Dodzuik, International Journal of Quantum Chemistry. 107(12), 2204(2007), DOI:10.1002/qua.21366

9. S. Grimme, C. Muck-Kichtenfled and J. Antony, Journal of Physical Chemistry C, 111(30), 11199(2007), DOI:10.1021/jp0720791

10. M. D. Curtis, J. Cao, J. W. Kampf, Journal of American Chemical Society, 126(13), 4318(2004), DOI: $10.1021 /$ ja0397916

11. I. Mancini, G. Guella, F. Pietra, C. Debitus and J. Waikedre, Helvetica Chimica Acta, 79(8), 2075 (1996), DOI: 10.1002/hlca.19960790804

12. H.R. Bokesch, L.K. Pannell, T.C. McKee and M.R. Boyd, Tetrahedron Letters, 41(33), 6305(2000), DOI: 10.1016/S0040-4039(00)01062-5

13. A. Casapullo, G. Bifulco, I. Bruno and R. Riccio, Journal of Natural Products, 63(4), 447(2000), DOI: $10.1021 / \mathrm{np} 9903292$ 
RASĀYAN J. Chem.

Vol. 13 | No. 1 |663 - 671| January - March | 2020

14. J. Sponer and P. Hobza, Chemical Physics Letters , 267(3), 263(1997), DOI:10.1016/S00092614(97)00118-8

15. S. Tsuzuki, K. Honda and R. Azumi, Journal of American Chemical Society, 124(41), 12200(2002), DOI: $10.1021 /$ ja0204877

16. B. K. Mishra and N. Sathyamurthy, Journal of Physical Chemistry A, 109(1), 6(2005), DOI: $10.1021 /$ jp045218c

17. B.K. Mishra and N. Sathyamurthy, Journal of Theoretical and Computational Chemistry, 5(3), 609(2006), DOI:10.1142/S0219633606002532

18. B.K. Mishra, J.S Arey and N. Sathyamurthy, Journal of Physical Chemistry A, 114(36), 9606(2010), DOI: $10.1021 / \mathrm{jp} 908941 \mathrm{u}$

19. M.G. Smith and A. Lemmerer, Journal of Molercular Structure, 1179, 132(2019), DOI: $10.1016 /$ j.molstruc.2018.10.038

20. M. J. Frisch, G.W. Trucks, H. B. Schlegel, P. M. W. Gill, B. G. Johnson, M. A. Robb, J. R. Cheeseman, T. Keith, G.A. Petersson, J.A. Montgomery, K. Raghavachari, M.A. Al-Laham, V. G. Zakrzewaki, J. V. Ortiz, J. B. Foresmann, J. Ciolowski, B. B. Stefanov, A. Namayakkara, M. Challacombe, C. Y. Peng, P. Y. Ayala, W. Chen, M. W. Wong, J. L. Andres, E. S. Replogle, R. Gomperts, R. L. Martin, D. J. Fox, B. J. S. inkley, D.J. Defrees, J. Baker, J. P. Stewart, M. HeadGordon, C. Gonzalez, and J.A. Pople, Gaussian (2009), Gaussian Inc., Pittsburgh PA.

[RJC-5535/2019] 